\title{
Erratum to: Study abroad programs as a service convergence: an international marketing approach
}

\author{
M. Minsuk Shin ${ }^{1} \cdot$ Eun Jeong Noh $^{2} \cdot$ Jiwon Lee $^{3}$
}

Published online: 11 May 2017

(C) Springer-Verlag Berlin Heidelberg 2017

\section{Erratum to: Serv Bus \\ DOI 10.1007/s11628-017-0345-6}

In the published study, the name of the authors are incorrect. The correct name of the authors is given in this erratum.

The original article was corrected.

The online version of the original article can be found under doi:10.1007/s11628-017-0345-6.

\author{
Jiwon Lee \\ yemannuer@khu.ac.kr \\ M. Minsuk Shin \\ shinm@konkuk.ac.kr \\ Eun Jeong Noh \\ eunjung0116@gmail.com
}

1 Department of International Trade, College of Commerce and Economics, Konkuk University, Sanghuh Hall \#638, 120 Neungdong-ro Gawngjin-gu, Seoul, South Korea

2 Research Institute for Social Criticality, Pusan National University, Busandaehak-ro 63beon-gil, Geumjeong-gu, Busan, South Korea

3 Department of International Business and Trade, College of Politics and Economics, Kyung Hee University, 26 Kyungheedae-ro, Dongdaemun-gu, Seoul, South Korea 